\title{
Downregulated Caveolin-1 expression in monocytes may contribute to the pathogenesis of psoriasis.
}

Naoko Takamura, Yukie Yamaguchi, Yuko Watanabe, Miho Asami, Noriko Komitsu and Michiko Aihara

Department of Environmental Immuno-Dermatology, Yokohama City University Graduate School of Medicine, Yokohama, Japan

\section{ABSTRACT}

【Background】 Caveolin-1 (Cav-1) is a membrane protein that is essential for the formation of flask-shaped membrane invaginations known as caveolae. Cav-1 regulates a variety of signaling molecules and receptors, and aberrant Cav-1 expression is involved in a variety of diseases. Our previous study revealed aberrant reduction of Cav-1 in the epidermis of psoriasis patients, which leads to enhanced phosphorylation of STAT3 and cytokine production, suggesting that decreased Cav-1 may contribute to psoriatic inflammation. Not only keratinocytes, but also immune cells are major players in psoriasis. Migrated immune cells produce a variety of effector cytokines which affect keratinocytes proliferation and induce psoriatic intlammation.

[Objectivel The purpose of this study was to determine whether Cav-1 is involved in the leucocyte function of psoriasis and investigate their role in the pathogenesis of psoriasis.

[Methods] We first determined Cav-1 expression levels in peripheral blood mononuclear cells (PBMCs) and polymorphonuclear cells (PMNs) isolated from 20 patients with psoriasis and 20 control subjects by quantitative PCR and immunoblotting. ic cell isolation were also conducted .

【Results】 mRNA and protein levels of Cav-1 in PBMCs and PMNs were significantly lower in psoriasis patients than control subjects. In PBMCs, significant reduction of Cav-1 was observed in CD14 positive monocytes. In addition we assessed function of Cav-1 decreased monocytes on cytokine production and migration. Cav-1 RNA interference in isolated monocytes resulted in elevated levels of IL-1 $1 \beta$ and IL-6 under LPS stimulation, compared to control monocytes. Furthermore, a migration assay revealed that Cav-1 silenced monocytes had enhanced chemotaxis activity induced by MCP-1.

【Conclusion】 Our results suggest that aberrant Cav-1 expression in monocytes may be involved in the pathogenesis of psoriasis by mediating cell migration and cytokine production.

\section{INTRODUCTION}

- Psoriasis vulgaris is a chronic immune-mediated inflammatory skin disease characterized by scaly papulosquamous plaque lesions (Griffiths and Barker, Lancet 370. 2007).

- The pathogenesis of psoriatic tissue hyperplasia is thought to be driven by the interplay of macrophages, dendritic cells (DCs), and pathogenic and resident memory $T$ cells, with enhanced representation of the IL-23-Th17/Th22 and IL-12-IFN-Y/TNF pathways (Lowes M. A. et al, Nature 445. 2007)

- Caveolin-1 (Cav-1) is a 22-kDa membrane protein essential for the formation of caveolae, which serve as a platform for processes such as lipid metabolism, endocytosis, signal transduction. Cav-1 down regulates signaling molecules and receptors, which interact with the Cav-1 scaffolding domain (CSD) corresponding to amino acids 82-101 of Cav-1.

\section{Our previous study}

We previously reported a significant reduction of Cav-1 in the epidermis of psoriasis patients.

A reduction of Cav-1 induces activation of the JAK/STAT pathway in psoriatic inflammation, which leads to further keratinocyte proliferation and cytokine/chemokine production.

CSD peptide interfered with psoriatic-

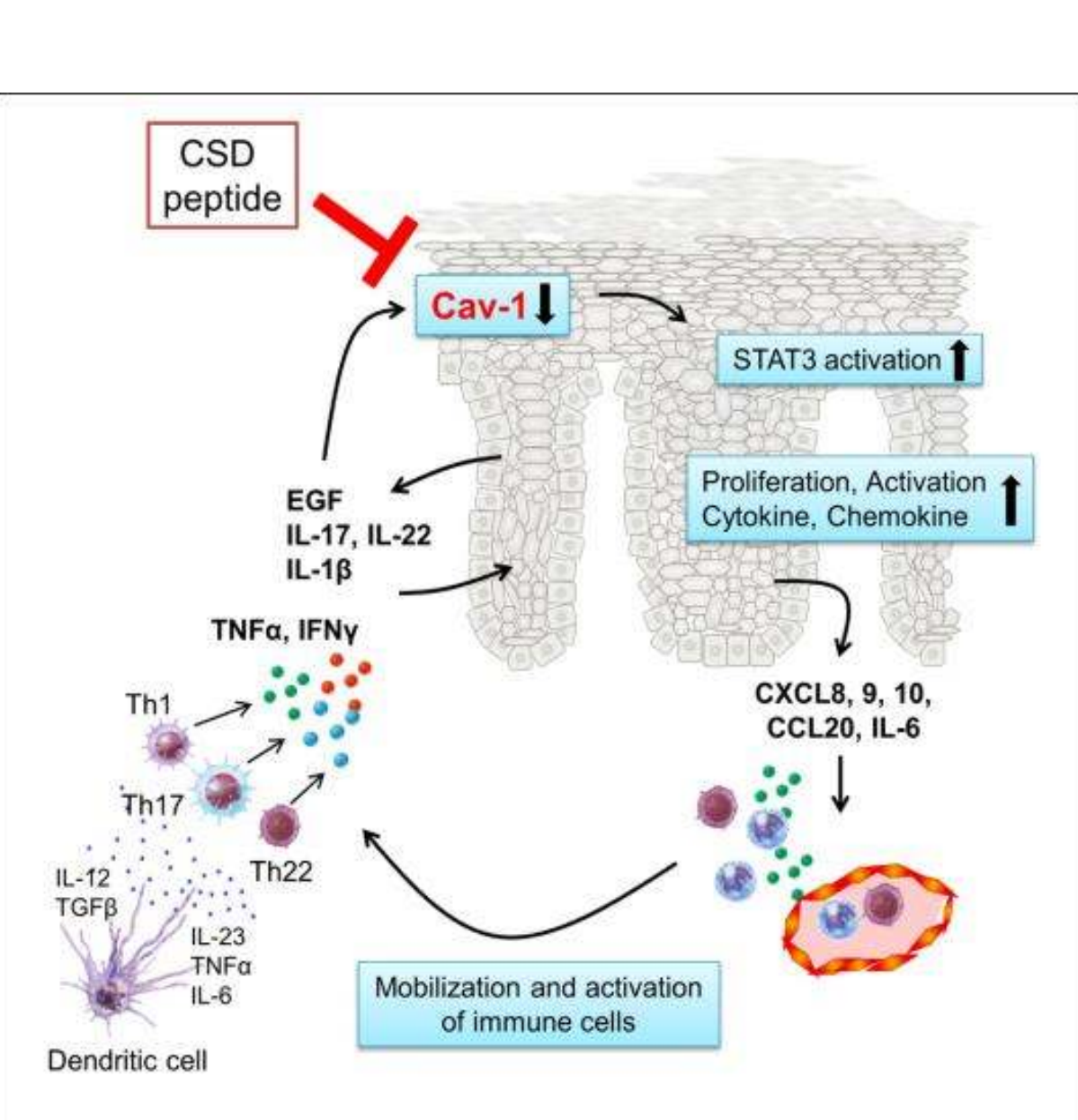
like skin inflammation in mice.

(Yamaguchi Y et al, J. Invest. Dermatol 135. 2015)

Less is known about the functions of Cav-1 in the cells of the immune system. Cav-1 is present in various classes of leucocytes, including monocytes/ macrophages, PMNs, mast cells and lymphocytes. It inhibits the expression of proinflammatory cytokines from macrophages by regulating the activation of mitogen-activated protein kinase (MAPK) family members.

\section{OBJECTIVE}

To examine whether Cav-1 is involved in the leucocytes function of psoriasis and investigate their role in the pathogenesis of psoriasis.

\section{METHODS \& RESULTS}

Decreased Cav-1 expression in the PBMCs and PMNs of psoriasis patients.

$>$ We first evaluated Cav-1 expressions in levels in peripheral blood mononuclear cells (PBMCs) and polymorphonuclear cells (PMNs) of 20 psoriasis patient (PSO) and 20 contro subjects $(\mathrm{HC})$. Samples were obtained after obtaining written informed consent, in accordance with the Declaration of Helsinki. The study was approved by the Institutional Review Board of Yokohama City University (Approval No: B120705021).

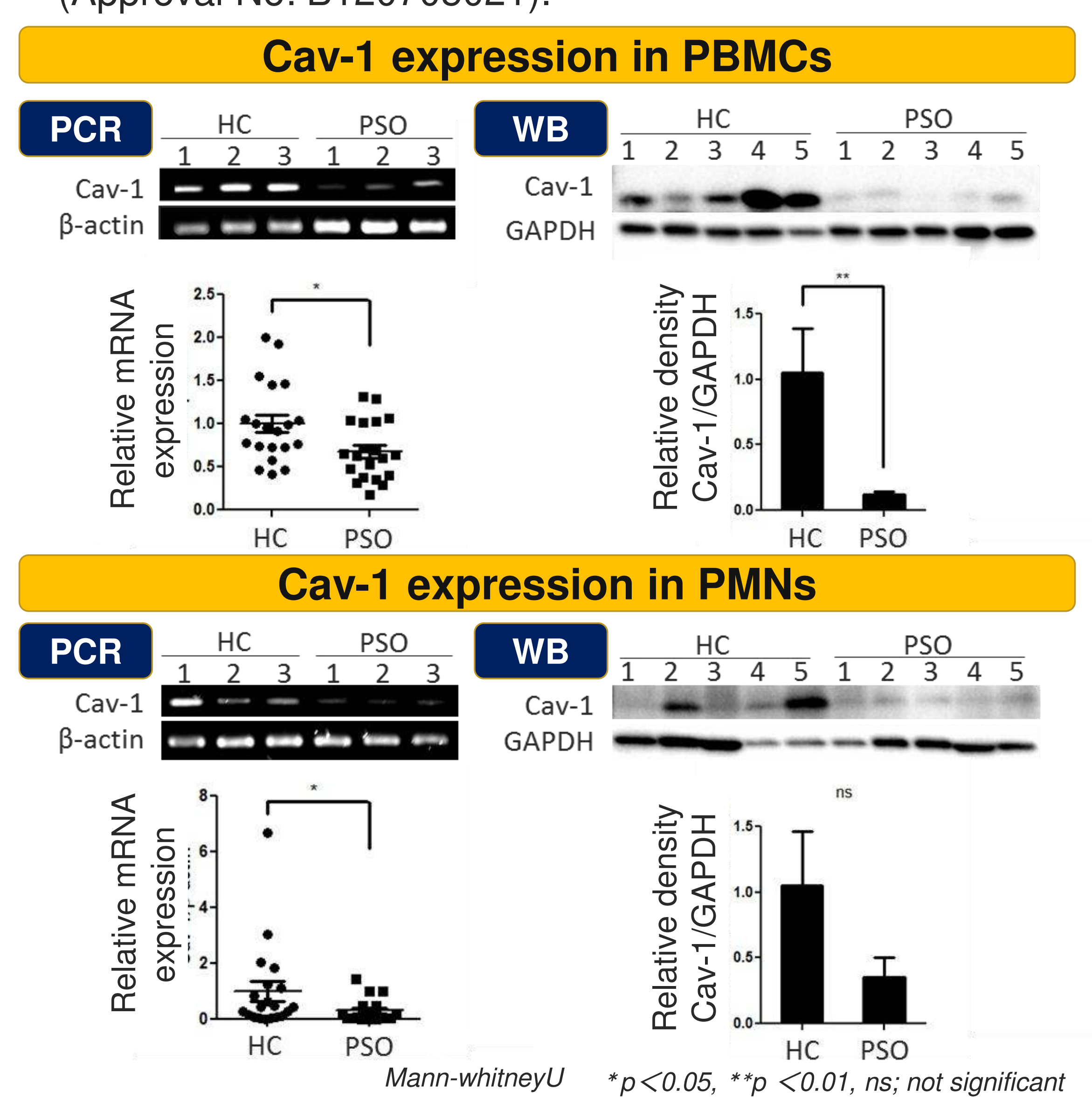

Cav-1 expression was found to be significantly reduced in PBMCs obtained from psoriasis patients by quantitative PCR (qPCR) and western blotting. Cav-1 reduction in PMNs of psoriasis patients was significant in mRNA levels but not in protein levels.

Decreased Cav-1 expression in circulating monocytes of psoriasis patients.

$>$ Cav- 1 expression levels in circulating $\mathrm{T}$ cells $\left(\mathrm{CD} 3^{+}\right)$, B cells (CD19+), and monocytes (CD14+) were evaluated by immunofluorescent microscopy.
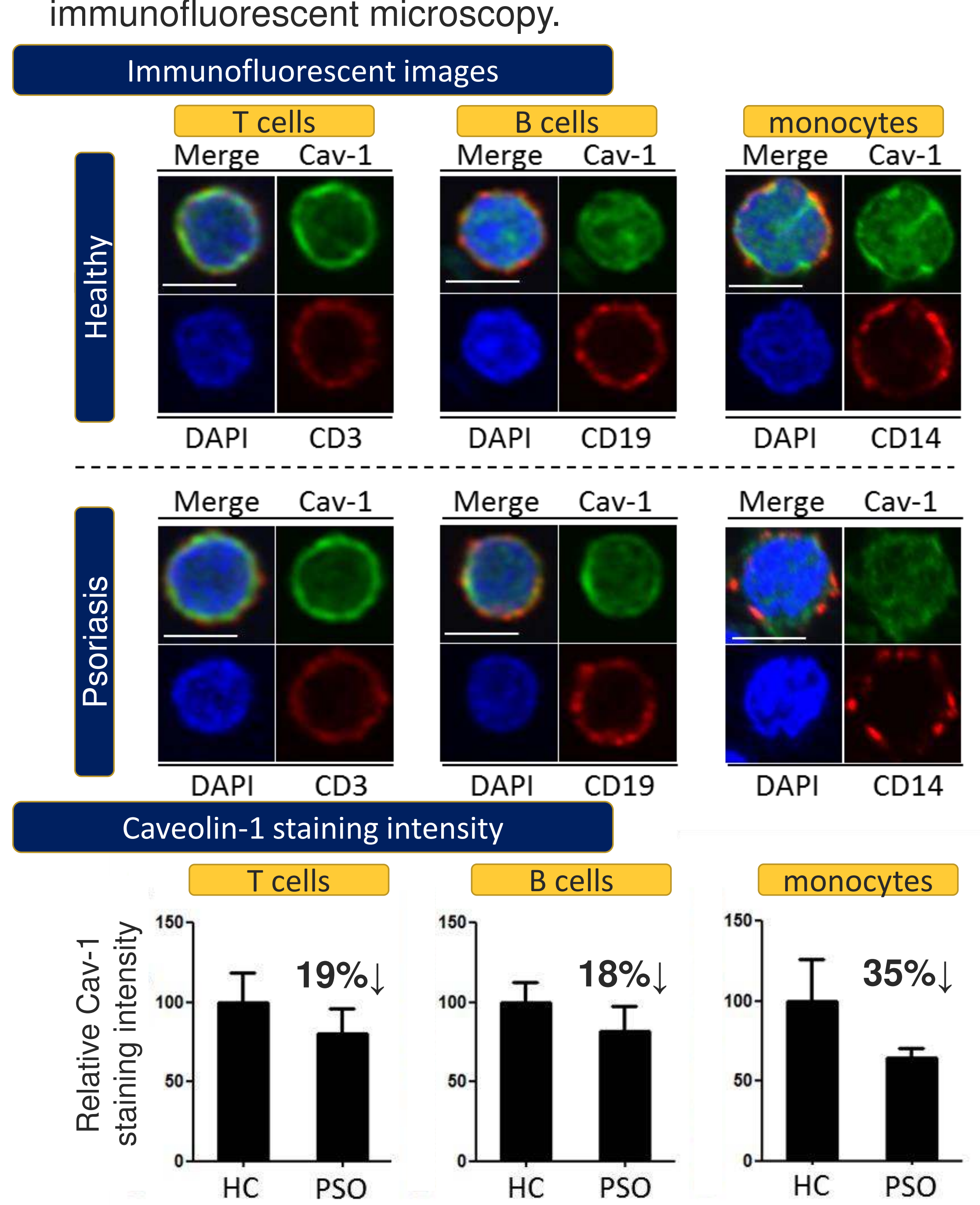

Cav-1 levels were decreased in T cells, B cells, and most prominent in monocytes.

\section{Cav-1 expression in Monocytes}

To confirm the reduction of Cav-1 levels in monocytes, monocytes were separated using MACS human monocyte isolation Kit II (Miltenyi Biotec, Bergisch Gladbach, Germany), and Cav-1 expression was examined by qPCR and western blotting
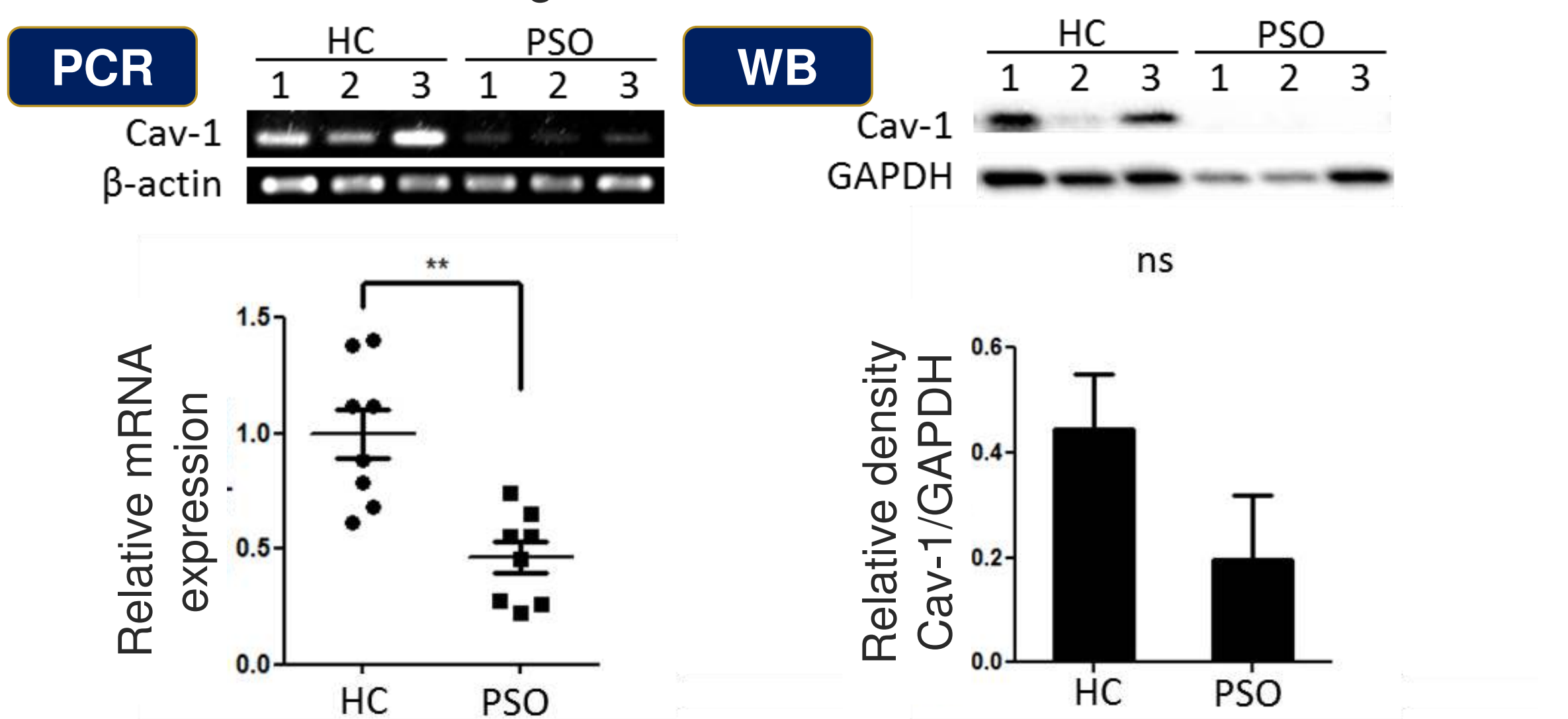

Cav-1 expression was significantly reduced in the monocytes of psoriasis patients.

\section{Hypothesis \\ Cav-1 downregulation in monocytes

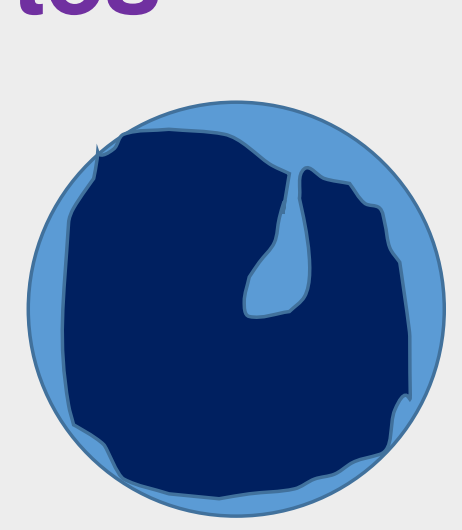 Cav-1 $\downarrow$ cytokines \\ $\underline{\text { Migration }}$ activities}

We evaluated whether downregulated Cav-1 in monocytes affected the production of inflammatory cytokines under LPS stimulation, or the migration activity toward MCP-1.

Enhanced cytokine production in Cav-1silenced monocyte

Human healthy monocytes were separated by MACS, and Cav-1 expression was silenced using sequence-specific siRNA. For transfection, Viromer Green (Lipocalyx GmbH, Halle (Saale), Germany) was used. Then, inflammatory cytokine levels induced by LPS were evaluated by qPCR

Cav-1-silenced or controlled monocytes
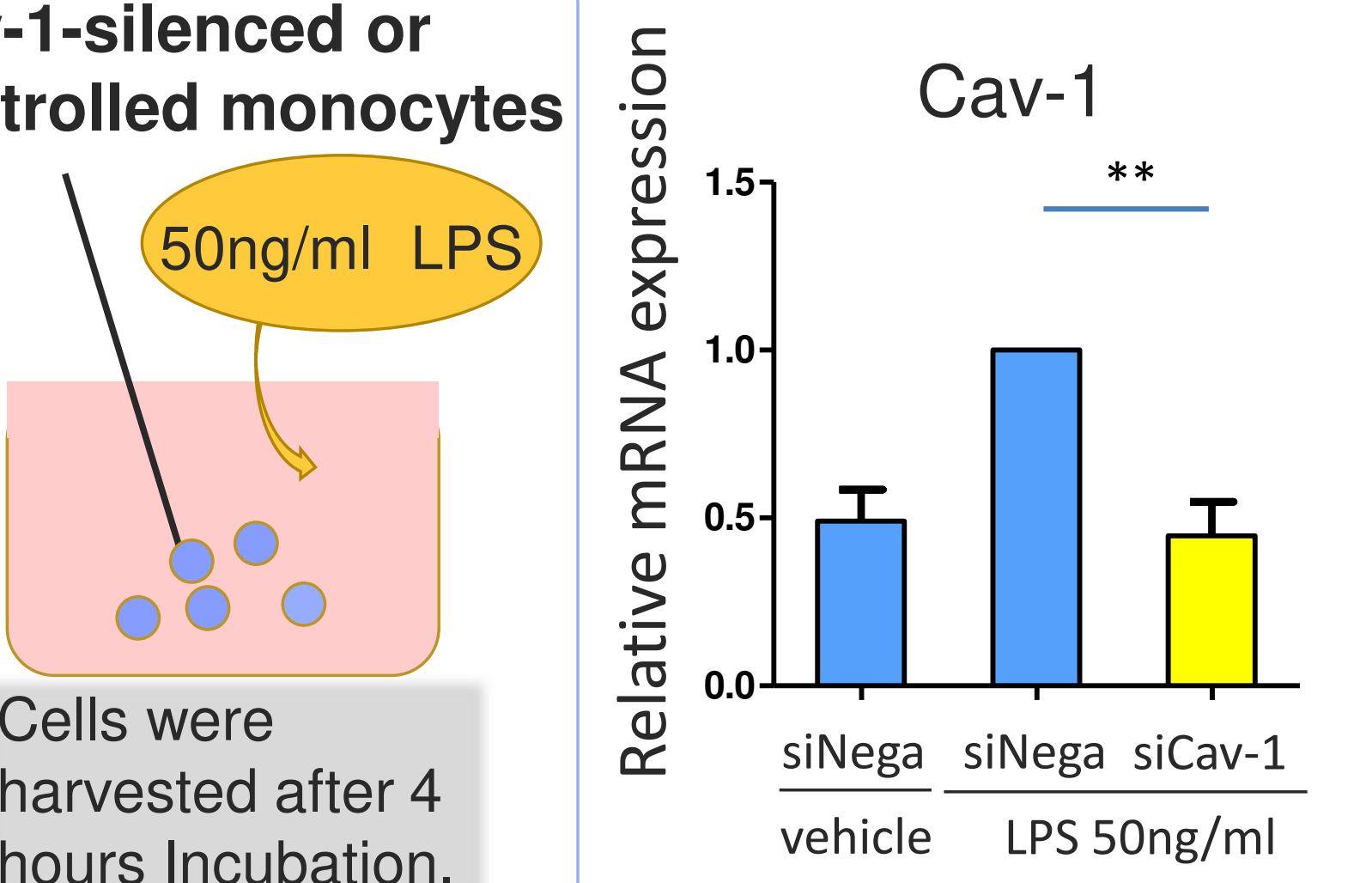

TNF- $\alpha$

hours Incubation
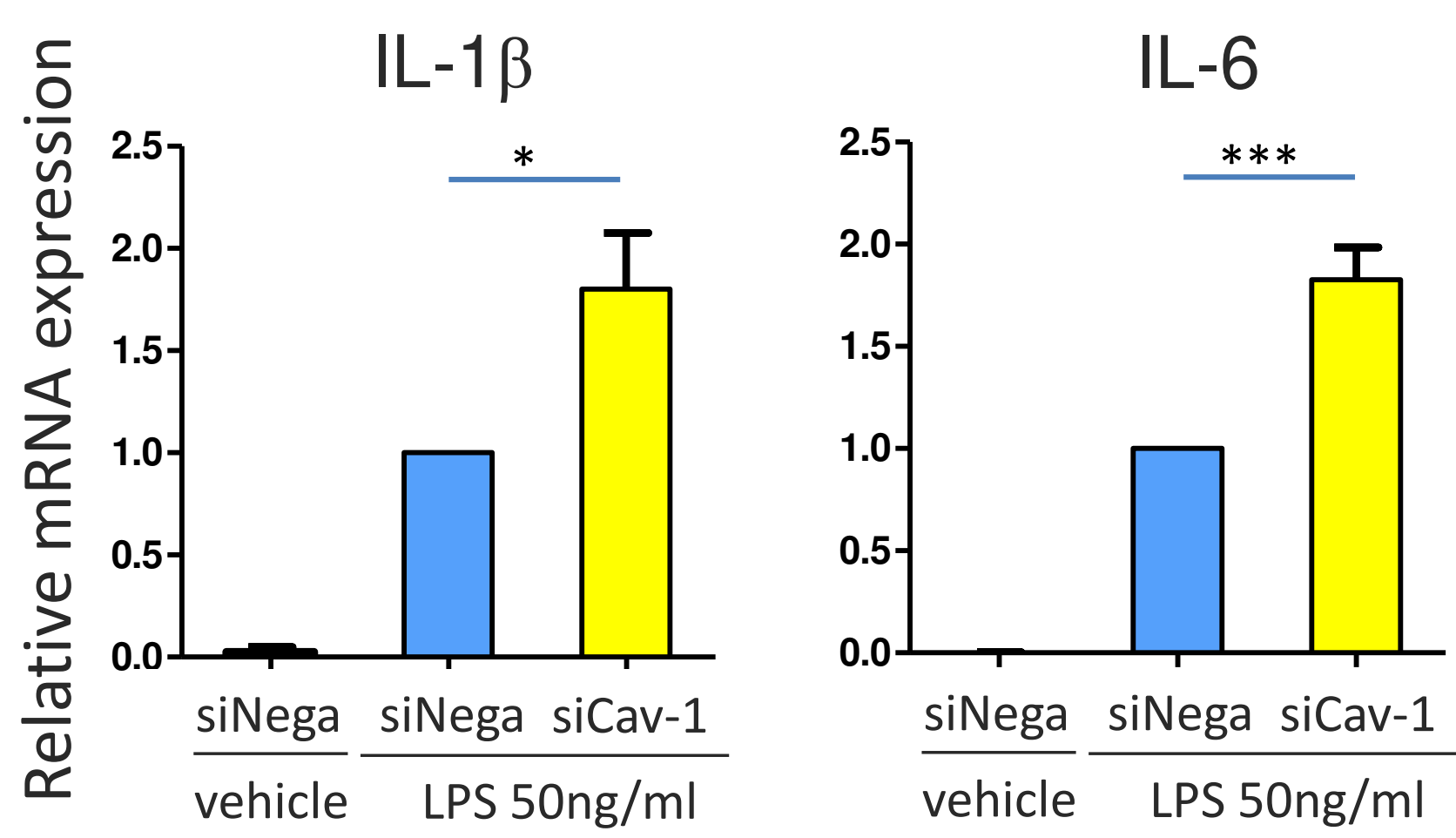

IL-10

Gene expression levels of IL- $1 \beta$ and IL- 6 in Cav-1silenced monocytes under LPS stimulation were significantly higher than that in controlled monocytes.

Enhanced migration activity in Cav-1silenced monocyte

The migration activity induced by MCP-1 of Cav-1-silenced or controlled monocytes was evaluated by migration assay. The percentage of migrated cells was calculated as a ratio of the cell count of the lower compartment to the total cell count of upper and lower compartments.

Cav-1-silenced or

controlled monocytes
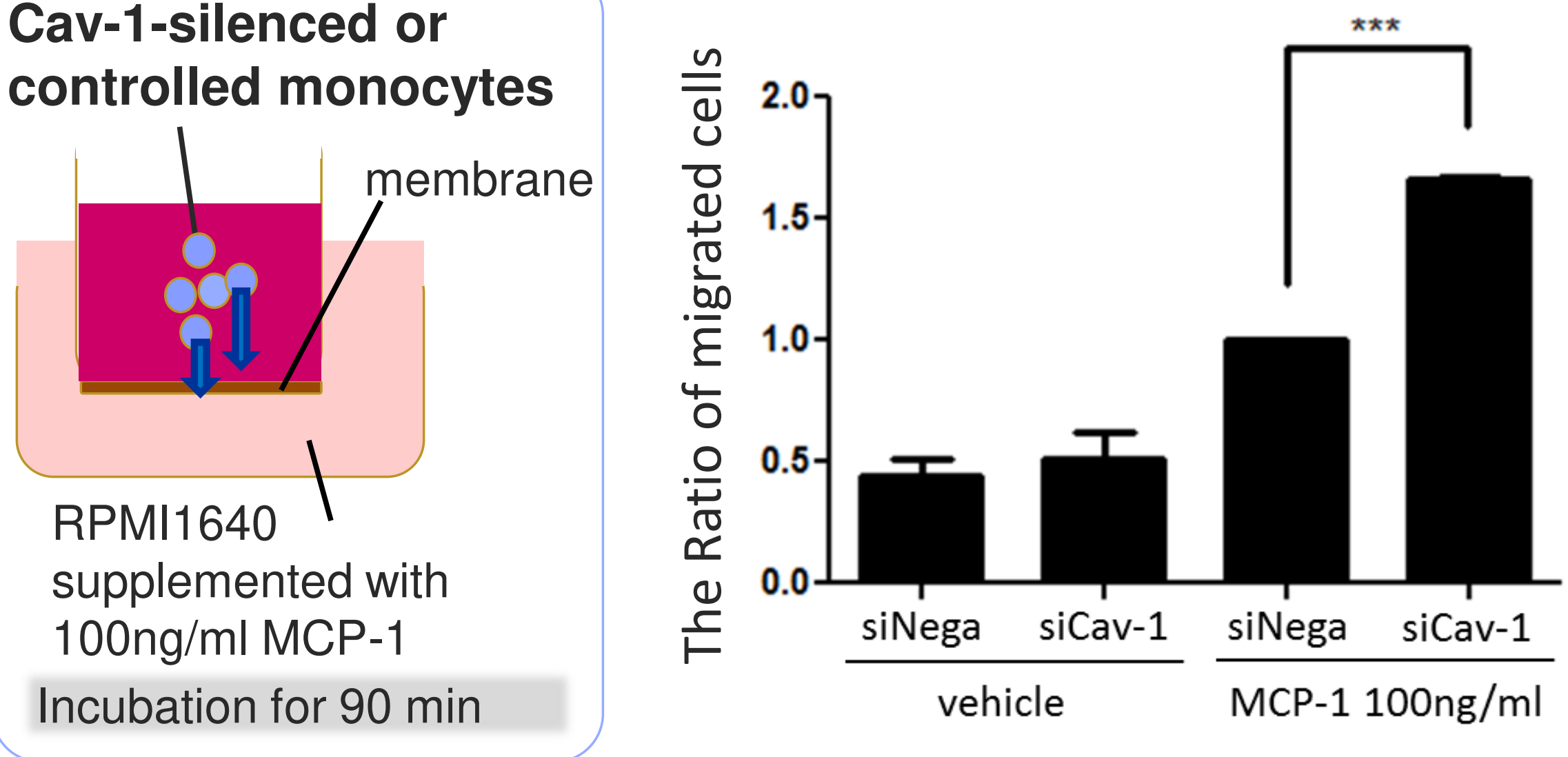

The ratio of migrated cells was significantly higher in Cav-1-silenced monocytes than that in controlled monocytes.

\section{SUMMARY}

1. Cav-1 expression is decreased in the PBMCs and PMNs of psoriasis patients.

2. Cav-1 expression is decreased in monocytes of psoriasis patients

3. Cav-1 reduction in monocytes enhances cytokine production and the migration activity.

\section{CONCLUSION}

Our results suggest aberrant Cav-1 expression on monocytes may be involved in the pathogenesis of psoriasis by mediating cytokine production and cell migration. 